\title{
Produção e captação de energia elétrica limpa através de adaptação de bicicletas ergométricas
}

\author{
Jeisiane Isabella da Silva Alexandre'*, Deivson Cesar Silva Sales², \\ João Pedro Ferreira Silva ${ }^{1}$, Ramon Borges Cordeiro ${ }^{1}$, Augusto Wagner \\ Viana Reis ${ }^{1}$
}

${ }^{1}$ Associação Caruaruense de Ensino Superior e Técnico, Av. Portugal, 584, Bairro Universitário, Caruaru-PE (CEP 55016-400). Estudante de Graduando em Engenharia Ambiental.*E-mail: jeisianebellas150@hotmail.com.

${ }^{2}$ Associação Caruaruense de Ensino Superior e Técnico, Av. Portugal, 584, Bairro Universitário, Caruaru-PE (CEP 55016-400). Professor Auxiliar I.

E-mail: deivsoncesar@yahoo.com.br.

Resumo. O crescimento da sociedade e o desenvolvimento de novas tecnologias implicaram no aumento da produção de equipamentos eletrônicos associado ao consumo de energia. Com a dominância da participação das fontes não renováveis na oferta mundial energética, presentes em quantidades limitadas, a sociedade busca por fontes alternativas de baixo custo e alta eficiência, como soluções para diminuição do impacto ambiental causado pelo aumento da poluição a nível mundial. A busca por energias renováveis de uso sustentável é a grande meta para o desenvolvimento de tecnologia mundial, preservando a Natureza. O presente trabalho apresentou como objetivo a construção e operação de um protótipo de equipamento para produzir e armazenar energia a partir de materiais recicláveis, pela adaptação das bicicletas ergométricas presentes em academias, para fins não comerciais. Foi utilizado um alternador automotivo ligado por uma corrente à roda da bicicleta ergométrica, cuja energia gerada foi armazenada em uma bateria do tipo ácido-chumbo. A montagem do equipamento foi realizada, onde foi observada a produção de energia em velocidades acima de $15 \mathrm{~km} / \mathrm{h}$, medida na própria bicicleta. Essa velocidade esteve relacionada com o número de giros no eixo do alternador. Nessas condições, o carregamento completo de um smartphone padrão foi realizado em $6 \mathrm{~h}$. Não foi necessária geração constante de energia para alimentar o sistema, apenas em intervalos de $40 \mathrm{~min}$ durante todo o processo. O carregamento completo do mesmo aparelho smartphone em na rede elétrica residencial de $220 \mathrm{~V}$, durou $4 \mathrm{~h}$.

Palavras-chave: Energia, Bicicleta ergométrica, Recicláveis.

Abstract. Production and catchment of clean electricity through the stationary bicycle adaptation. The growth of society and the development of new technologies resulted in increased production of electronic equipment associated with energy consumption. With the dominance of the participation of non-renewable sources in the world energy supply, limited quantities at present, the society search for alternative sources of low cost and high efficiency, as solutions for reducing the environmental impact caused by increased pollution worldwide. The search for renewable energy sustainable use is the

Recebido: 09/05/2015

Aceito: 12/06/2015

Publicado: 30/06/2015

Acesso Aberto Artigo completo

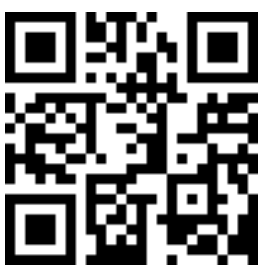


major goal for the development of global technology, preserving nature. This work presented as objective the construction and operation of a system prototype to produce and store energy from recyclable materials, the adaptation of stationary bikes present at gyms, for non-commercial purposes. An automotive alternator connected by a chain round the stationary bike, whose energy generated is stored in a battery type lead acid was used. The mounting was realized, where it was observed energy output at speeds above $15 \mathrm{~km} / \mathrm{h}$, measured at own bike. This speed was related to the number of turns on the alternator axis. Under these conditions, the full loading of a standard smartphone was accomplished in $6 \mathrm{~h}$. There was no need for constant generation of power supply to feed the system, only at intervals of 40 min throughout the procedure. Fully charging the same smartphone device in the residential electrical network of $220 \mathrm{~V}$ lasted $4 \mathrm{~h}$.

Keywords: Energy, Stationary bicycle, Recyclable.

\section{Introdução}

Devido ao crescimento da sociedade e constante desenvolvimento de novas tecnologias, houve aumento na produção de equipamentos eletrônicos, associado a um maior consumo de energia elétrica. Esse aumento da demanda energética implicou na escassez dos recursos naturais e grandes impactos ambientais, devido à necessidade de expansão da produção hidrelétrica ou aumento no consumo de combustíveis fósseis (Bronzatti e Iarozinski Neto, 2008). Nesse sentido, surge a necessidade de alternativas de fontes energéticas de baixo custo e alta eficiência (Jannuzzi, 2000).

Os métodos alternativos de produção de energia devem ter caráter renovável e buscar a sustentabilidade sem impactos ao meio ambiente (Moreira e Cardoso, 2010). Dessa forma, o desenvolvimento das ditas "tecnologias verdes" é atrativo para se alcançar esses objetivos (Huang e Rust, 2011). Essas tecnologias visam o reaproveitamento de materiais que se apresentaram como rejeitos de outros processos produtivos, através da aplicação de métodos como a reciclagem ou o reuso. Aplicação desses materiais classificados como rejeitos, para a produção de energia elétrica usada no carregamento de equipamentos eletrônicos pessoais, tais como celulares, atendem às propostas da sustentabilidade ambiental (Strzelecki et al., 2007).
Outro ponto de destaque nessa atual sociedade é a busca por uma maior qualidade de vida. Essa qualidade de vida está intimamente associada à prática de atividades físicas (Strzelecki et al., 2007). Nas academias, o uso de equipamentos eletronicos (principalmente os smartphones) já divide espaço com essa prática cotidiana de exercícios físicos, devido à necessidade pessoal de se manter sempre conectado às diversas atividades diárias relativas ao trabalho, redes sociais, entre outros (Oikawa, 2013). Devido ao intenso uso, esses equipamentos precisam ser recarregados diversas vezes, ocasionando um consumo excessivo de energia elétrica (Strzelecki et al., 2007).

No sentido de associar a produção de energia elétrica para carregamento de equipamentos eletrônicos (principalmente os smartphones) à qualidade de vida através das atividades físicas, tendo em vista também a sustentabilidade ambiental, no presente trabalho foi avaliada a adaptação de uma bicicleta ergométrica para geração de energia, a partir de materiais recicláveis descartados como rejeitos, para fins não comerciais.

\section{Materiais e métodos}

\section{Materiais}

Na produção de energia elétrica por meio da adaptação de uma bicicleta ergométrica, foi usado um alternador 


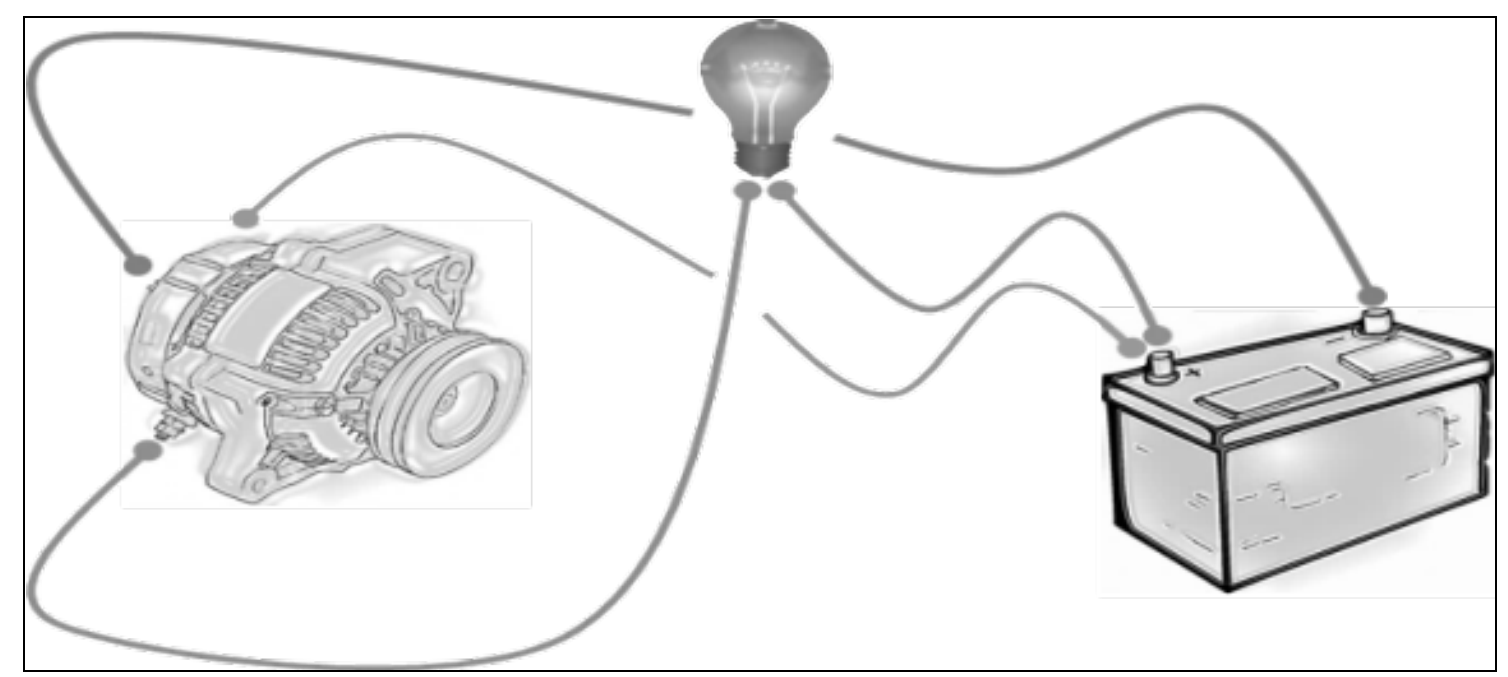

Figura 1. Esquema de montagem do sistema adaptado para captação de energia usando um alternador automotivo.

automotivo (6 cc; $138 \mathrm{cv} ; 90 \mathrm{~A})$; uma corrente acoplada à roda da bicicleta ergométrica, adaptada com uma catraca; bateria de motocicleta (ácido-chumbo; 12,0 V; $7 \mathrm{Ah}$; 2,25 kg); conversores de tensão de $12,0 \mathrm{~V}$ para $5,0 \mathrm{~V}$, adaptado de acendedores de cigarro automotivos, a partir de entradas do tipo Universal Serial Bus (USB); recipiente formado de MDF (medium-density fibreboard), onde o equipamento foi devidamente isolado e montado.

\section{Métodos}

Tendo em vista a produção de energia elétrica para carregamento de smartphones, através da adaptação de bicicletas ergométricas usadas em academias, o presente trabalho fez uso de materiais do tipo recicláveis. $\mathrm{O}$ equipamento foi construído com base no princípio da indução eletromagnética (Halliday et al., 2010), a partir do qual uma corrente induzida foi gerada pela rotação de um alternador automotivo (6 cc; $138 \mathrm{cv}$; 90 A). A geração da corrente esteve limitada ao movimento de rotação do rotor do alternador, ligado por uma corrente à roda da bicicleta ergométrica adaptada com uma catraca. A energia gerada foi armazenada em uma bateria de motocicleta (bateria do tipo ácido-chumbo; 12,0 V; $7 \mathrm{Ah} ; 2,25 \mathrm{~kg}$ ), conforme o esquema apresentado na Figura 1.
Para o carregamento dos smartphones, foi associado ao sistema da bateria um conversor de tensão de $12,0 \mathrm{~V}$ para 5,0 V, adaptado de acendedores de cigarro automotivos, a partir de entradas do tipo USB. O equipamento foi devidamente isolado e montado em um recipiente formado de MDF (medium-density fibreboard), descartado da fabricação de móveis. O sistema de baixo custo teve em vistas as aplicações do tipo não comerciais, tendo todos os equipamentos sido adquiridos por doação, pois seriam descartados. Na sequência, foi avaliada a eficiência de carregamento de um smartphone (1,2 GHz Quad Core; Adreno 305; bateria de lítio; $2.070 \mathrm{MAh}$ ) em termos do tempo de carregamento, em comparação com o carregamento em rede elétrica residencial convencional.

\section{Resultados e discussão}

A adaptação entre o alternador e a roda da bicicleta ergométrica encontra-se apresentada na Figura 2. No sistema, a catraca que permitiu o giro do alternador foi ligada à roda da bicicleta por uma corrente. O movimento de giro da roda fez com que a catraca girasse e movimentasse 0 eixo do alternador.

$\mathrm{O}$ uso de um alternador que gera corrente contínua é anexado às rodas da bicicleta ergométrica, criando energia 


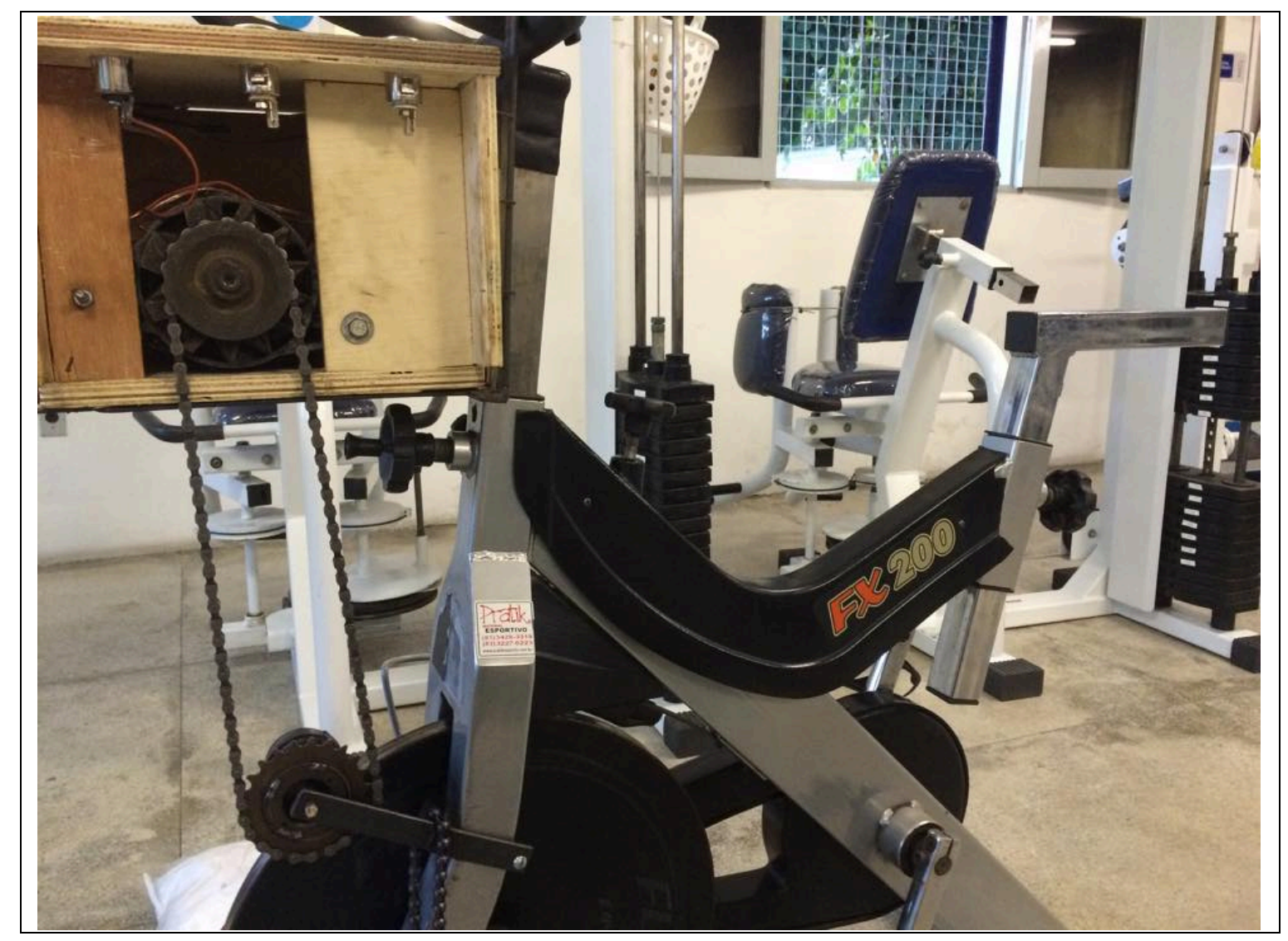

Figura 2. Sistema adaptado para captação de energia usando um alternador automotivo.

elétrica durante os giros forçados. Esse equipamento é responsável por conversão da energia mecânica em elétrica, e induz essa corrente elétrica em um condutor por meio de um campo magnético, que posteriormente será armazenada em uma bateria fechada comum do tipo ácidochumbo, na qual três componentes participam da reação eletroquímica: placa positiva (dióxido de chumbo - $\mathrm{PbO}_{2}$ ), placa negativa (chumbo puro esponjoso - $\mathrm{Pb}$ ) e ácido sulfúrico $\left(\mathrm{H}_{2} \mathrm{SO}_{4}\right)$, que fornece íons de hidrogênio e sulfato.

As entradas do tipo USB foram ligadas à bateria, nos polos positivo e negativo, respectivamente, conforme a Figura 3.

Essa ligação por cabo via porta USB permitiu a conversão entre a tensão cedida pela bateria e a necessária ao carregamento dos smartphones. Através de medição da tensão, foi constado que a geração de energia ocorreu quando a velocidade média (medida na própria bicicleta) foi superior a $15 \mathrm{~km} / \mathrm{h}$. Essa velocidade esteve relacionada com o número de giros proporcionados pela catraca ao alternador. Nessas condições, a lâmpada que promovia a indução do alternador se apagava. Na sequência, o smartphone descarregado foi posto sob condição de carregamento no sistema (Figura 4) até carga máxima, que foi alcançada após duração de aproximadamente $6 \mathrm{~h}$.

O novo carregamento da bateria para manter o smartphone carregando durante as $6 \mathrm{~h}$ só foi necessário quando a ela começava a diminuir a tensão fornecida abaixo de 7,0 V (abaixo dessa tensão não ocorria o carregamento), o que durava cerca de $40 \mathrm{~min}$. Não foi necessário manter o movimento de giro do alternador de forma ininterrupta durante todo tempo de carga, apenas quando essa tensão se tornava inferior aos 7,0 V. O carregamento completo desse mesmo aparelho usando a tensão residencial de $220 \mathrm{~V}$ foi alcançado em aproximadamente $4 \mathrm{~h}$. 


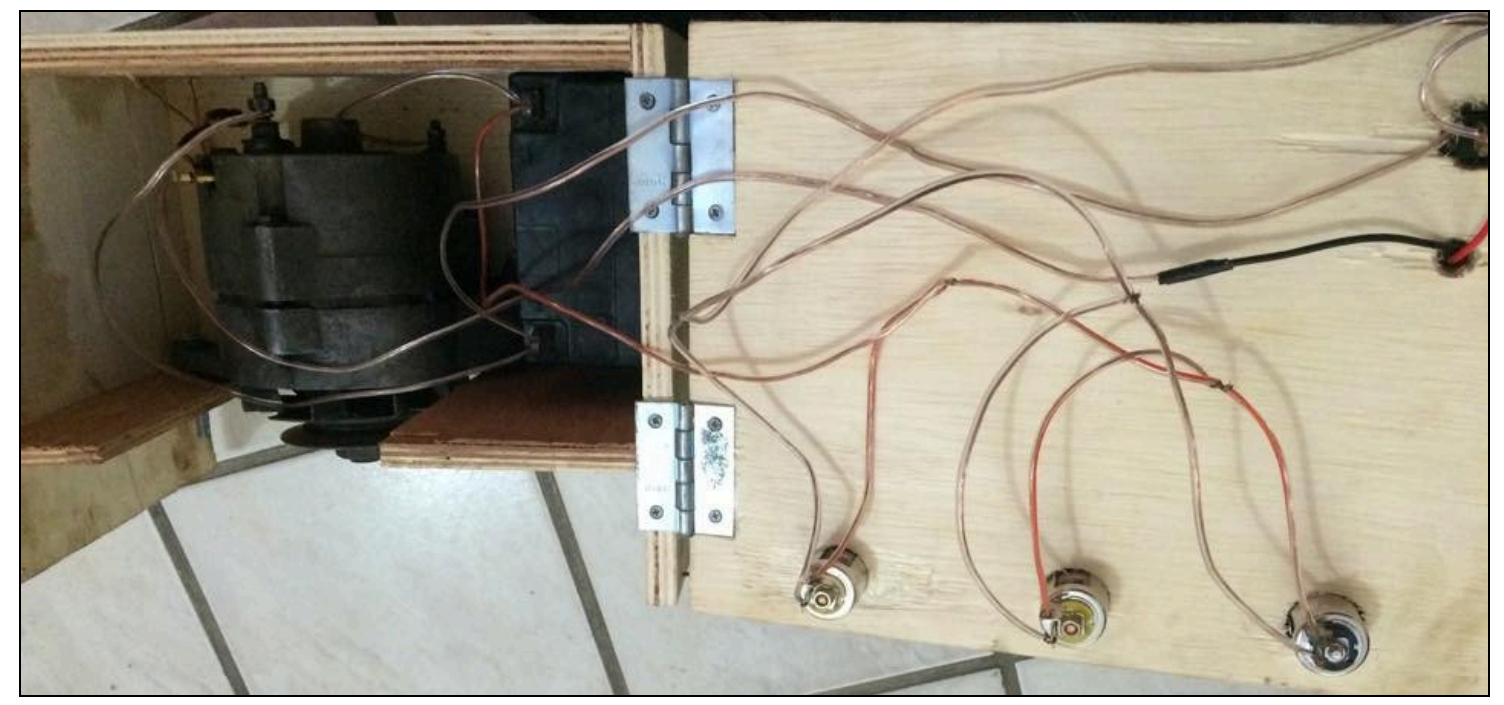

Figura 3. Ligação entre as entradas USB e a bateria.

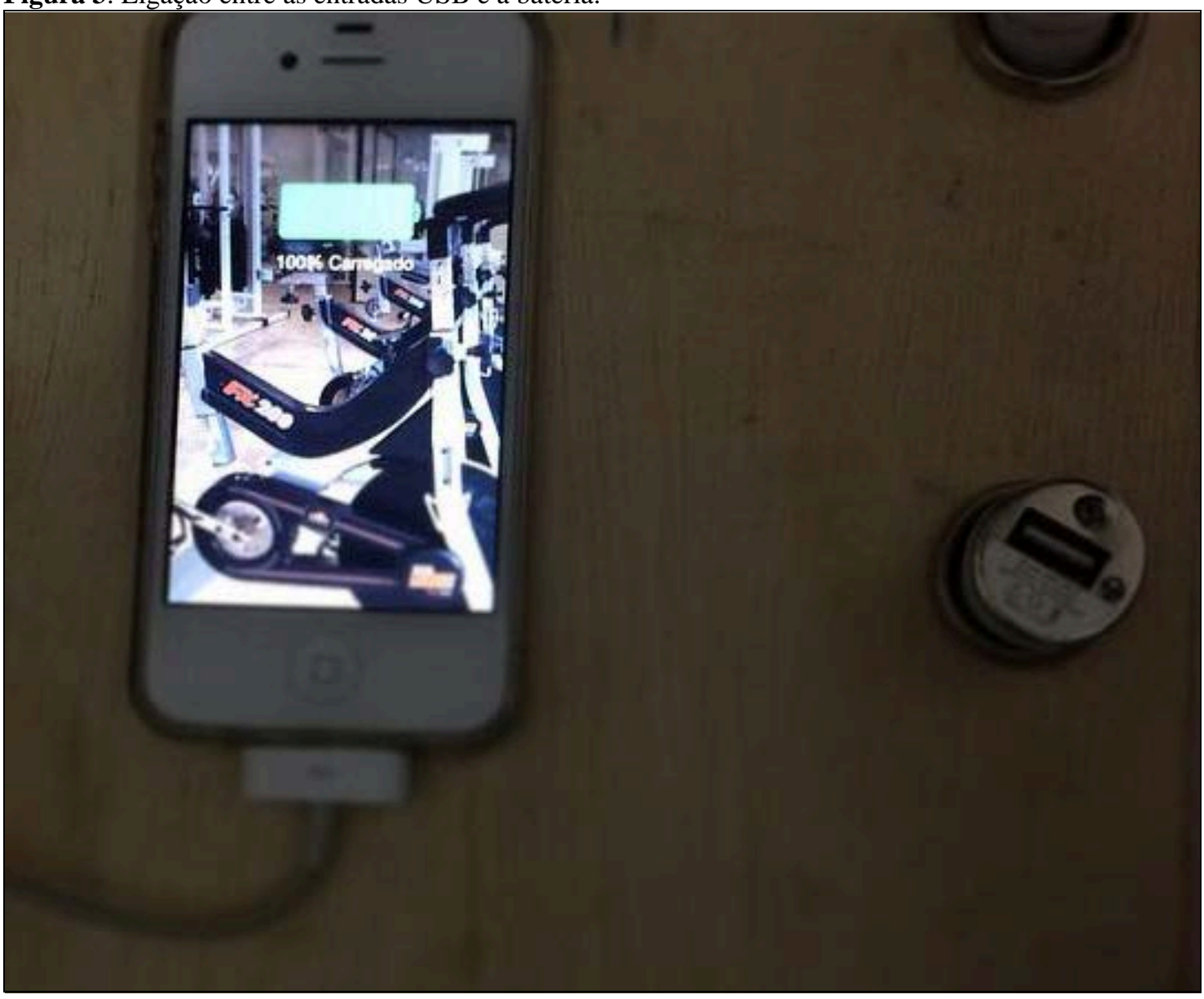

Figura 4. Carregamento do aparelho smartphone pelo sistema.

\section{Conclusão}

No presente trabalho, materiais recicláveis foram utilizados para desenvolver um protótipo de sistema de carregamento de equipamentos eletrônicos, por adaptação de bicicletas ergométricas. É um sistema de geração que não agride o meio ambiente e ao mesmo tempo proporciona uma atividade física para $\mathbf{0}$ atleta que visa cuidar da sua saúde, além de ser uma forma de geração de baixo custo. 
É possível a adaptação de uma bicicleta estacionária para que converta energia mecânica em elétrica e sua aplicação prática seria na utilização em academias com bicicletas ergométricas adaptadas para a transformação de energia. Explorando-se esse conceito, entende-se que o ponto fundamental do uso das energias alternativas é encontrar formas sustentáveis e limpas de converter essas energias entre si. Portanto, trata-se de um projeto que visa, além de demonstrar o fenômeno da transformação de energia, uma forma de obtenção de energia elétrica sustentável e limpa associada a uma redução de gasto de energia no local.

\section{Referências}

Bronzatti, F. L.; Iarozinski Neto, A. Matrizes energéticas no Brasil: Cenário 2010-2030. Encontro Nacional de Engenharia de Produção, 2008. v. 28.

Halliday, D.; Resnick, R.; Walker, J. Fundamentals of physics extended. New York: John Wiley \& Sons, 2010.
Huang, M.-H.; Rust, R. T. Sustainability and consumption. Journal of the Academy of Marketing Science, v. 39, n. 1, p. 40-54, 2011.

Jannuzzi, G. M. Políticas públicas para eficiência energética e energia renovável no novo contexto de mercado: uma análise da experiência recente dos EUA e do Brasil. São Paulo: Autores Associados/Fapesp, 2000.

Moreira, C. E. S.; Cardoso, A. M. Fontes alternativas de energia renovável, que possibilitam a prevenção do meio ambiente. Bolsista de Valor, v. 1, n. 1, p. 397-402, 2010.

Oikawa, E. Os paradoxos hipermodernos e as tecnologias digitais: reflexões sobre a sociabilidade contemporânea a partir das práticas de "bem-estar". Sessões do Imaginário, v. 18, n. 30, p. 89-96, 2013. Disponível em: <http://revistaseletronicas. pucrs.br/ojs/index.php/famecos/article/view/145 08/11076>. Acesso em: 14 jan. 2015.

Strzelecki, R.; Jarnut, M.; Benysek, G. Exercise bike powered electric generator for fitness club appliances. In: Power Electronics and Applications, European Conference on, IEEE, p. 1-8, 2007. 